\title{
Estimation of $500 \mathrm{kV}$ power transmission line parameters with PMU
}

\author{
Yuri Kononov*, Evgenii Zelenskii, Olga Rybasova, Dmitrii Kostyukov, \\ Ekaterina Bakaushina \\ North Caucasian Federal University, Russia, Stavropol
}

\begin{abstract}
This work is devoted to the research of the real-time estimation of $500 \mathrm{kV}$ power transmission lines parameters with PMU based on the solution of the optimization problem of minimizing the root-mean-square deviations of the specific line parameters or the sum of the modules of the correlation coefficients between the specific parameters and the sum of the squares of the voltages or currents measured at the ends of the line. Independent optimization variables are the correction factors of the measuring systems of currents and voltages. Based on an artificially simulated PMU dataset with specified Gaussian noise and systematic errors, it is shown that the use of correlation coefficients in the objective function is more effective than standard deviations. All 5 estimated coefficients turned out to be closer to the reference values. The results of calculations are obtained from the data of real PMUs for an operating 500 $\mathrm{kV}$ line with a length of $504.6 \mathrm{~km}$. The deviation of the specific capacitive conductivity from the nominal value is $0.13 \%$, compared with $-0.29 \%$ when using the sum of squares of deviations as an objective function.
\end{abstract}

\section{Introduction}

One of the important tasks of the modern power system in conditions of high variability of power flow is to reduce losses of electrical energy in electrical networks while ensuring the required level of reliability. An effective measure for solving this problem in electric networks of extra-high voltage (EHV) (330 kV and above) is the optimal control of powerfactor correction units and voltage regulation devices. The relevance of a practical solution to this problem will increase as the networks are saturated with modern FACTS devices of various types, installed both at substations and directly on long lines [1].

To solve the problem of optimizing the power flow in terms of voltage and reactive power, it is necessary to have an up-to-date and accurate model of the power. Usually, for these purposes, $\pi$-equivalent circuit for transmission lines are used, determined by the geometric characteristics and physical properties of wires, considering their parameters unchanged [2].

However, it is known that the dependence of specific corona losses is nonlinear on voltage. Also, due to the reactive corona effect, the capacitive conductivity of the

\footnotetext{
* Corresponding author: iukononov@ncfu.ru
} 
transmission line increases with an increase in corona losses [3]. In good weather and, accordingly, low corona losses, the assumption under consideration does not lead to practically significant errors in the control of the power flows. However, in bad weather conditions, corona losses increase by 1-2 orders of magnitude, which can lead to an increase in the errors of the received control actions and economic damage due to use of simplified EHV transmission lines model, based on the equation of a long line and a refined $\pi$-equivalent circuit [3].

Setting the parameters of the lines according to the nominal data also does not take into account changes that occur during operation (pulling wires, aging insulators), and the effect of weather conditions (first of all, temperature on the resistance and ice on the capacitive conductivity).

On-line estimation of power lines parameters with PMU under certain conditions can give more accurate results than determining by design and geometric characteristics.

At the moment, there are several dozen researches in which equations and algorithms for estimation the parameters of the power lines $\pi$-equivalent according to PMU data are proposed [4-10].

In these researches, various assumptions are made and methods for estimation the parameters of power transmission line models are considered. They are differ in taking into account the asymmetry, wave properties of the line, the influence of parallel circuits, the presence of taps, and a number of other factors.

It is not difficult to show [18] that if we have got the vectors (complexes) of voltages and currents of the positive sequence of the fundamental harmonic from the PMU, installed at the ends of the transmission line, for any $i$-th moment in time, the parameters of the $\pi$ equivalent circuit can be determined as:

$$
\begin{gathered}
\boldsymbol{Y}^{i} / 2=G^{i} / 2-j \cdot B^{i} / 2=\frac{\mathbf{I}_{1}^{i}-\mathbf{I}_{2}^{i}}{\mathbf{U}_{1}^{i}+\mathbf{U}_{2}^{i}} \\
\boldsymbol{Z}^{i}=R^{i}+j \cdot X^{i}=\frac{\mathbf{U}_{1}^{i}-\mathbf{U}_{2}^{i}}{\mathbf{I}_{1}^{i}-\left(\mathbf{Y}^{i} / 2\right) \cdot \mathbf{U}_{2}^{i}}
\end{gathered}
$$

where $\boldsymbol{Y}^{\boldsymbol{i}}$ - is complex conductivity of the line, including active conductivity $G^{i}$ and capacitive conductivity $B^{i}$, equally divided in the $\pi$-equivalent circuit;

$\boldsymbol{Z}^{\boldsymbol{i}}$ - is complex resistance of the $\pi$-equivalent circuit of the line, including active $R^{i}$ and inductive $X^{i}$ resistance;

$\mathbf{I}_{1}^{i}, \mathbf{I}_{2}^{i}$ - are complexes (vectors) of positive sequence currents at the beginning and the end of the line ;

$\mathbf{U}_{1}^{i}, \mathbf{U}_{2}^{i}-$ are complexes (vectors) of positive sequence voltage at the beginning and at the end of the line.

Obviously, the accuracy of the estimation of the $\pi$-equivalent circuit parameters according to formulas (1) and (2) will depend on the inaccuracy of current and voltage transformers and the PMU. Thus the considered problem is related to the compensation of systematic errors and reduce the influence of random (noise) error measuring systems. The fact is that measuring current and voltage transformers installed at substations, as a rule, are not calibrated during operation due to the high required costs. In addition, their inaccuracy depends on the actual current, voltage and a number of other factors (temperature, degree of deterioration, etc.).

To solve this problem, researchers have proposed different ways. An analysis of its current state is given in the article [16]. The authors denote three main approaches to reducing the errors of measuring systems:

1. The statistical approach based on processing a large number of measurements from SCADA systems taking into account the topology of the network circuit, Kirchhoff's current law, common bus voltage magnitude etc. 
2. Using accurate meters at critical locations and then calibrating remaining meters by extended state estimation.

3. Joint solution to the problem of estimation of parameters of transmission lines and calibration of measuring devices.

Unfortunately, despite a lot of works that have appeared in the last two decades and are devoted to this issue, the problem of improving the accuracy of estimation the lines parameters remains relevant because of the high sensitivity of these parameters to measurement errors. As the authors of [16] note, in order to improve the accuracy of estimation of the lines parameters, it is necessary to simultaneously solve the problem of determining the systematic errors of measuring systems installed at the ends of the lines. Another authors [15] argue that at the time of this writing, they know only one work [13], devoted to the simultaneous determination of the lines parameters and systematic errors of measuring systems. The authors has developed an approach based on the nonlinear optimization, for the identification of ratio correction factors (RCFS) by minimizing the sum of squared residuals between calculated $\pi$-equivalent circuit parameters and it estimated values. In their next work [14] the authors provide to supplement the objective function with weighting mechanisms for each component of the residual function. A similar approach was proposed by the authors of this article to estimation the parameters of the $750 \mathrm{kV}$ transmission line of the UES of Russia in 2015 [12] and developed for the $500 \mathrm{kV}$ transmission line in [4]. However, in contrast [14], the correlation coefficient between the estimated parameters and the sum of the squares of the current at the ends of the line was taken as the objective function [4]. Due to differences in objective functions, the results of estimating the transmission lines parameters may differ, which requires additional research to compare them.

In this paper, Section 2 describes the methodology and algorithm of the research program for the simultaneous estimation of transmission line (TL) parameters and RCFS from PMU based on the optimization of a flexible objective function that includes both standard deviations and correlation coefficients. Section 3 contains the characteristics of the investigated $500 \mathrm{kV}$ lines and their power flows, and the research plan.

The results of the studies performed are given in Section 4. This section contains an analysis of the root-mean-square deviations and errors of the estimated parameters of the transmission line in the presence of Gaussian noise in the measurements of currents and voltages and the absence of systematic errors.

It also analyzes the results of estimation the line parameters and RCFS for $500 \mathrm{kV}$ transmission line with different composition of the objective function, both from artificially noisy measurements with specified systematic errors, and from real PMU.

\section{Methodology and algorithm}

The proposed method for estimation the transmission lines parameters is based on the approach suggested in $[12,4]$. It is based on the following assumptions:

1 . The parameters of systematic errors are taken unchanged over an identifiable time interval.

2. Random errors of voltage and current measuring systems have a normal distribution law.

3. The parameters of the power flow (currents and voltages) on the identified time interval have fluctuations that exceed by an order of magnitude the random errors of the measuring systems.

4. $\pi$-equivalent circuit of positive sequence is used for modelling power transmission lines. 
Let us denote the coefficients for compensating the systematic error (or ratio correction factors, RCFS) of currents and voltages of the positive sequence at the beginning and end of the line by the following symbols: $K_{U 1}, K_{\delta 1}, K_{I 1}, K_{\varphi 1}, K_{U 2}, K_{\delta 2}, K_{I 2}, K_{\varphi 2}$. Then expressions (1) and (2) for estimating the line parameters can be written as follows:

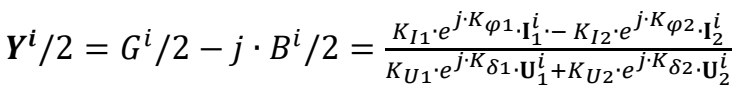

$$
\begin{aligned}
& \boldsymbol{Z}^{i}=R^{i}+j \cdot X^{i}=\frac{K_{U 1} \cdot e^{j \cdot K_{\delta 1} \cdot \mathbf{U}_{1}^{i}-K_{U 2} \cdot e^{j \cdot K} \delta 2 \cdot \mathbf{U}_{2}^{i}}}{K_{I 1} \cdot e^{j \cdot K_{\varphi 1}} \cdot \mathbf{I}_{1}^{i}-K_{U 2} \cdot e^{j \cdot K} \delta 2 \cdot\left(\mathbf{Y}^{i} / 2\right) \cdot \mathbf{U}_{2}^{i}}
\end{aligned}
$$

In general, these coefficients are found by minimizing the objective function:

$$
\begin{gathered}
f\left(K_{U 1}, K_{\delta 1}, K_{I 1}, K_{\varphi 1}, K_{U 2}, K_{\delta 2}, K_{I 2}, K_{\varphi 2}\right) \\
=\sum_{P}\left(\mu_{P} C V(P)+H(P)\right)+\sum_{V} \sum_{W} \mu_{V W} \cdot|C O R(V, W)| \rightarrow \min ,
\end{gathered}
$$

where $P \in\left\{R, X, G, B, R_{0}, X_{0}, G_{0}, B_{0}\right\}$ - a set of line parameter, including specific active resistance $R_{0}$, specific inductive resistance $X_{0}$, specific active conductivity $G_{0}$ and specific capacitive conductivity $B_{0}$ taken conventionally considered constant at the time interval $\mathrm{T}$, which is assumed to be known for the vector of currents and voltages for each $i$-th moment time;

$C V(P)$ - mean - square deviations of the $P$ parameter, calculated in accordance with (3)-(4) on the identified time interval $T$ for each $i$-th moment in time, relative to their respective mean values in this interval;

$\mu_{p}-$ is the weighting factor of each component of the standard deviation in the objective function;

$\operatorname{COR}(V, W)$ - is the correlation coefficient between a pair of parameters $\mathrm{V}, \mathrm{W}$ :

$V \in P \cup\left\{U_{1}^{2}+U_{2}^{2}, I_{1}^{2}+I_{2}^{2}, \delta_{1}-\delta_{2}, \delta_{1}-\varphi_{1}, \delta_{2}-\varphi_{2}\right\}$,

$W \in P \cup\left\{U_{1}^{2}+U_{2}^{2}, I_{1}^{2}+I_{2}^{2}, \delta_{1}-\delta_{2}, \delta_{1}-\varphi_{1}, \delta_{2}-\varphi_{2}\right\}$,

$\mu_{V W}$ - is the weighting factor of each correlation coefficient in the objective function;

$H(P)$ - is the penalty function to take into account the constraints on the estimated $P$ parameter;

$$
H(P)=\left\{\begin{array}{lc}
0, & P_{\text {min }} \leq P \leq P_{\text {max }} \\
K_{1} \cdot\left(P_{\text {min }}-P\right)^{K_{2},} & P<P_{\text {min }}, \\
K_{1} \cdot\left(P_{\text {max }}-P\right)^{K_{2},} & P>P_{\text {max }}
\end{array}\right.
$$

where $K_{1}, K_{2}-$ is the weighting factor,

$P_{\min }, P_{\max }$ - are the maximum permissible values of the appropriate parameter of the power transmission line.

The formulated problem refers to a nonlinear programming problem with constraints in the form of equalities (3)-(4), imposed on independent and dependent optimization variables, and inequalities (6), imposed on dependent optimization variables. The independent variables are the coefficients RCFS, bounded on two sides.

To find the minimum of a function, it is possible to use the coordinate descent method, the simulated annealing method, the Monte Carlo method, or a combination of these, or any other algorithm for solving similar problems. In the present work, a combined method was used, including the use of the method of enumeration over a given grid at the first stage and the method of coordinate descent at the second stage. This method was implemented in a computer program specially developed for research, written in the $\mathrm{C}++$ language.

The main problem is to choose the optimal composition of the objective function. In this paper, were proposed the following assumptions when choosing an objective function:

$1 . \pi$-equivalent circuit is reduced and takes into account the distribution of parameters. It is known [22], that in the equivalent circuit, each of the parameters $(R, X, G, B)$ depends on all of the specific parameters $\left(R_{0}, X_{0}, G_{0}, B_{0}\right)$. To solve the inverse problem, i.e. 
determination of specific parameters $\left(R_{0}, X_{0}, G_{0}, B_{0}\right)$ based on the parameters of the $\pi$ equivalent circuit, while developing the program algorithm, the calculated expressions given in [17] were used. It should be noted that this approach requires knowledge of the exact value of the line length. As rightly noted in the work [16], modern geodetic tools allow to solve such a problem with a minimum error.

2. Software algorithm provides the possibility of calculating equivalent vectors positive sequence currents and voltages with PMU for any amount $N T_{1}$ measurements, a multiple number of all measurements at the interval $\mathrm{T}$. It also provides for the possibility of decimating PMU measurements and averaging the calculated parameters in accordance with (3)-(4) for the $i$-th point in time in order to reduce the impact on the error of the results. Averaging is carried out for a given amount $N T_{2}$ calculated in accordance with (3)(4) parameter values. Averaged over small time intervals parameters $R^{i}, X^{i}, G^{i}, B^{i}$ ire used to calculate $R_{0}^{i}, X_{0}^{i}, G_{0}^{i}, B_{0}^{i}$ and constituting an objective function (5)-(6).

3. Especially for research, pseudo-measurements are formed with specified noise characteristics and systematic errors as close as possible to the power flow of a real line based on real PMU installed at the ends of the transmission line. For this, the values of measurements of currents and voltages of one of the ends of the line are taken as conditionally reference and according to the nominal parameters of the line and Ohm's law equations, conditionally reference vectors of currents and voltages of the other end of the line are calculated. Then the conditional reference values are corrected in accordance with the specified systematic errors, which are superimposed by the required noise level.

4. The program algorithm provides for various service functions:

- maintaining a database of nominal parameters of lines, set limits and initial approximations for dependent and independent optimization variables, objective function coefficients (5)-(6) for each line;

- the possibility of customizable logging of the optimization process;

- saving the results of estimation of the line parameters and RCFS in csv format.

To evaluate the efficiency of the proposed in papers $[12,4]$ algorithm based on minimizing the correlation coefficient $\operatorname{COR}(V, W)$ in comparison with an algorithm based on minimizing the standard deviation $C V(P)$, proposed in [14], as well as development proposals for further simultaneous estimation algorithms improve line parameters and RCFS was held numerical experiments according to the PMU, installed at the ends of the current of $500 \mathrm{kV}$, located in southern Russia UES .

\section{Case study}

Studies were carried out on the operated line $500 \mathrm{kV}$. The length of it is $504.643 \mathrm{~km}$. Each phase of the line is made in the form of three steel-aluminum wires AC-300/66, fixed along the tops of an equilateral triangle with spacers at a distance of $40 \mathrm{~cm}$. According to the passport data, the line has the following specific nominal parameters: $R_{o}=0.0373 \mathrm{Ohm} / \mathrm{km}, X_{0}=0.3042 \mathrm{Ohm} / \mathrm{km}, B_{o}=3.8946 \mu \mathrm{S} / \mathrm{km}, G_{0}=0.012275 \mu \mathrm{S} / \mathrm{km}$. Recalculated based accounting distributivity $\pi$-equivalent circuits: $R=16.941 \mathrm{ohm}$, $X=146.0247$ ohms, $B / 2=1008.13 \mu \mathrm{S}, G / 2=6.48 \mu \mathrm{S}$.

According to the ends of the line set WAMS: on the one side - PMU «MIP-01», produced by «RTSoft» company, PMU «RP-411» produced by «Parma » company on the other side. PMU connected to the measuring current transformer and voltage transformer with accuracy figure 0.2 . These devices measure in real time every $20 \mathrm{~ms}$ and transmit to the data concentrator the values of the voltage and current vectors for the positive sequence, as well as the frequency, active and reactive power. For the study, measurements were used for November 30, 2020 in the period from 12:00 to 13:00. According to data from 
meteorological services, in the settlements near which the line runs, the air temperature during this period averaged about $5 \mathrm{C}$, high humidity, and light rain some places.

The research was carried out using the developed computer research program in two stages.

At the first stage, was used mathematical modeling based on the measurement data of currents $I_{1}$ and voltages $U_{1}$ at the beginning of the line in accordance with clause 3 given in Section II of this article. Pseudo- measurements of currents and voltages with specified values of systematic errors of measuring systems and Gaussian noise were formed.

By pseudo - measurements was estimated line parameters and their standard deviations and variation coefficients while the values RCFS is proper. And then impact analysis value Gaussian noise on the accuracy of the parameter estimation was held.

The influence was evaluated for three variants of the magnitude of the Gaussian noise.

Then, based on pseudo - measurements were performed two experiments differing composition used objective function for simultaneously estimation line parameters and RCFS. In the first experiment, the standard deviations of the estimated line parameters were used as the objective function. In the second, the sum of the modules of the correlation coefficients $\operatorname{COR}(V, W)$ were used as the objective function.

At the second stage, real measurements from the PMU were used without preliminary processing, «as is». The nominal parameters of the line were used as a conditional standard to assess the effectiveness of the proposed method.

While choosing independent variables of optimization and influencing the results of the tuning coefficients of the program, the following assumptions were made:

1. The amount of parameters to be optimized has been reduced from 8 to 5 . This is due to the fact that research has shown [14] optimum values of coefficients $K_{U 1}$ and $K_{U 2}, K_{\delta 1}$ and $K_{\delta 2}, K_{I I}$ and $K_{I 2}, K_{\varphi 1}$ and $K_{\varphi 2}$ are mutually bonded to each other. In this regard, when setting all 8 coefficients as optimization variables, the minimum of the objective function (5) is not unambiguous and pronounced. Therefore, in the studies, the coefficients $K_{U 2}, K_{I 2}$, $K_{\varphi 1}, K_{\varphi 2}, K_{\delta 2}$ were taken as independent variables of optimization.

2. The weighting factors $\mu_{p}$ are chosen so that the deviation of each of the parameters had similar effects on the objective function: $\mu_{R}=0.0590284, \mu_{x}=0.00684814$, $\mu_{G}=0.0771664, \mu_{B}=0.000495969$.

3. From physical laws known that the values of the parameters $X_{0}, B_{0}$ is weakly dependent from the power flow, temperature and other weather phenomena. At the same time, the active resistance linearly depends on the temperature of the wires, the overheating of which depends on the current flowing through them. In addition, in previously published works [19-21] it is shown that corona losses depend on stresses in the power of 4-10. In addition, there is a reactive effect of the corona [20]. Therefore, when using the objective function $\operatorname{COR}(V, W)$ as components, the weighting factors were taken equal to zero at the correlation coefficients between: $R_{0}$ and $I_{1}^{2}+I_{2}^{2}, G_{0}$ and $U_{1}^{2}+U_{2}^{2}, B_{0}$ and $U_{1}^{2}+U_{2}^{2}$.

\section{Results}

\subsection{Study of the root-mean-square deviations and errors of the estimated line parameters in the presence of Gaussian noise in the measurements of currents and voltages}

Measurements of current and voltage vectors from PMU differ from their real values due to the presence of systematic and random errors introduced by measuring current and voltage 
transformers, their secondary circuits, limited bits of ADC and PMU controller, inaccuracies in their synchronization, shortcomings of signal processing algorithms, conducted noise and many of other factors [10]. Random errors can be maximally compensated for by appropriate calibration of the PMU [10] or in the SCADA. It is very difficult to completely avoid and compensate for random errors. Therefore, even if full compensate systematic errors in measurement systems, there are errors in estimation results due to the presence of random errors, also known in the theory of measuring Gaussian noise.

The influence of random errors to the problem of estimation of the line parameters can be reduced by a method known from the theory of measurements, based on averaging the parameters estimated in accordance with (1)-(2).

The studies were performed for three combinations of random errors:

1) values as close as possible to real ones and given in [14]: $0.03 \%$ for voltages and $0.04 \%$ for currents, 0.017189 degrees for their phase angles (related to a synchronization accuracy of $1 \mu \mathrm{s}$ ).

2) doubled voltage and current variations.

3) 5 times reduced phase angle variations (which related to a synchronization accuracy of $200 \mathrm{~ns})$.

The results of calculating the root-mean-square deviations and errors of the estimated parameters of the investigated $500 \mathrm{kV}$ transmission line are shown in Table $1\left(P_{N}\right.$ is the nominal value of the parameter).

Table 1. Study of the effect of random errors on estimated line parameters at full compensation of systematic errors in measuring currents and voltages

\begin{tabular}{|c|c|c|c|c|c|c|c|c|}
\hline Parameter & $\begin{array}{c}\text { R, } \\
\text { Ohm }\end{array}$ & $\begin{array}{c}\mathrm{X}, \\
\text { Ohm }\end{array}$ & $\begin{array}{l}\mathrm{G}, \\
\mu \mathrm{S}\end{array}$ & $\begin{array}{l}\mathrm{B}, \\
\mu \mathrm{S}\end{array}$ & $\begin{array}{c}\text { R0, } \\
\text { Ohm / } \\
\text { km }\end{array}$ & $\begin{array}{c}\text { X0, } \\
\text { Ohm / } \\
\text { km }\end{array}$ & $\begin{array}{c}\text { G0, } \\
\mu \mathrm{S} / \mathrm{km}\end{array}$ & $\begin{array}{c}\text { B0, } \\
\mu \mathrm{S} / \mathrm{km}\end{array}$ \\
\hline \multicolumn{9}{|c|}{ At $\sigma(\mathrm{U})=0,03 \%, \sigma(\mathrm{I})=0,04 \%, \sigma(\delta)=\sigma(\varphi)=0,017189^{\circ}$} \\
\hline $\bar{P}$ & 16,940 & 146,024 & 12,957 & 2016,26 & 0,037 & 0,304 & 0,012 & 3,895 \\
\hline$\sigma(P)$ & 0,305 & 0,308 & 0,910 & 0,885 & 0,001 & 0,001 & 0,002 & 0,002 \\
\hline$\frac{\sigma(P)}{\bar{P}} \cdot 100, \%$ & 1,803 & 0,211 & 7,025 & 0,044 & 1,798 & 0,222 & 14,039 & 0,043 \\
\hline$\frac{\left(\bar{P}-P_{N}\right)}{P_{N}} \cdot 100, \%$ & $-0,004$ & 0,000 & $-0,018$ & 0,000 & $-0,004$ & 0,000 & $-0,031$ & 0,000 \\
\hline \multicolumn{9}{|c|}{ At $\sigma(U)=0,06 \%, \sigma(I)=0,08 \%, \sigma(\delta)=\sigma(\varphi)=0,017189^{\circ}$} \\
\hline $\bar{P}$ & 16,940 & 146,024 & 12,957 & 2016,26 & 0,037 & 0,304 & 0,012 & 3,895 \\
\hline$\sigma(P)$ & 0,596 & 0,339 & 1,477 & 1,543 & 0,001 & 0,001 & 0,003 & 0,003 \\
\hline$\frac{\sigma(P)}{\bar{P}} \cdot 100, \%$ & 3,519 & 0,232 & 11,398 & 0,077 & 3,505 & 0,246 & 22,505 & 0,076 \\
\hline$\frac{\left(\bar{P}-P_{N}\right)}{P_{N}} \cdot 100, \%$ & $-0,008$ & 0,000 & $-0,019$ & 0,000 & $-0,008$ & 0,000 & $-0,029$ & 0,000 \\
\hline \multicolumn{9}{|c|}{ At $\sigma(U)=0,06 \%, \sigma(I)=0,08 \%, \sigma(\delta)=\sigma(\varphi)=0,0034377^{\circ}$} \\
\hline $\bar{P}$ & 16,940 & 146,025 & 12,959 & 2016,256 & 0,037 & 0,304 & 0,012 & 3,895 \\
\hline$\sigma(P)$ & 0,296 & 0,102 & 0,683 & 0,737 & 0,001 & 0,000 & 0,001 & 0,001 \\
\hline$\frac{\sigma(P)}{\bar{P}} \cdot 100, \%$ & 0,017 & 0,001 & 0,053 & 0,000 & 0,017 & 0,001 & 0,103 & 0,000 \\
\hline$\frac{\left(\bar{P}-P_{N}\right)}{P_{N}} \cdot 100, \%$ & $-0,004$ & 0,000 & $-0,004$ & 0,000 & $-0,004$ & 0,000 & $-0,005$ & 0,000 \\
\hline
\end{tabular}

Analysis of the results leads to the following conclusions:

1. An increase in Gaussian noise in measurements of signal amplitudes leads to a significant increase in the standard deviations of the estimated parameters and a less significant increase in the deviation from the nominal, which is explained by the reference nature of the simulated Gaussian noise. Gaussian noise has the greatest effect on active conductivity, then resistance and the smallest on capacitive conductivity. Obviously, for given levels of Gaussian noise errors in measurements of voltages and currents and for real 
variations in the $\pi$-equivalent circuits parameters, there is an optimal value of the interval over which it is necessary to average the estimated parameters.

2. Increasing the Gaussian noise in measurements of signal amplitudes by two times has the most negative effect on the standard deviation of the inductive reactance (it increases from $0.07 \%$ to $0.21 \%$ ). This phenomenon can be explained by the fact that the difference in phase angles of voltages has the greatest effect on the value of the estimated inductive resistance.

3. The smallest variation at the investigated levels of Gaussian noise has the capacitive conductance. This allows us to assume that the weighting factor in the objective function proposed in [14], with capacitive conductance $B_{0}$ should be maximum.

\subsection{The results of estimation of parameters and RCFS for $500 \mathrm{kV}$ line in the presence of specified systematic errors}

Most authors [14-16] dealing with the problem of estimating the parameters of lines suggest using as an objective function the sum of the squares of the deviation of the estimated parameters from their mean values or the standard deviations calculated on their basis. In the opinion of the authors of this work, this leads to an overestimation of the average value of the estimated parameter.

In this paper we propose use correlation coefficients between specific parameters $R_{o}, X_{o}, G_{o}, B_{o}$ and the sum of squares of the currents and voltages .

For comparison of these two approaches to the reference measurements of the systematic errors are added: $U_{1}=-0.1 \%, U_{2}=+0.05 \%, I_{1}=+0.08 \%, I_{2}=+0,12 \%, \delta_{1}=0^{\circ}$, $\delta_{2}=+0.2^{\circ}, \varphi_{1}=-0.4^{\circ}, \varphi 2=-0.2^{\circ}$.

Tables 2 and 3 show the results of a comparison of the two approaches.

Table 2. The results of estimation of line parameters within using the sum of squares of deviations of the estimated line parameters as an objective function

\begin{tabular}{|c|c|c|c|c|c|c|c|c|}
\hline Parameter & $\begin{array}{c}\boldsymbol{R}, \\
\mathbf{O h m}\end{array}$ & $\begin{array}{c}\boldsymbol{X}, \\
\mathbf{O h m}\end{array}$ & $\begin{array}{c}\boldsymbol{G}, \\
\boldsymbol{\mu} \mathbf{S}\end{array}$ & $\begin{array}{c}\boldsymbol{B}, \\
\boldsymbol{\mu} \mathbf{S}\end{array}$ & $\begin{array}{c}\boldsymbol{R}_{\mathbf{0}}, \\
\mathbf{O h m} / \\
\mathbf{k m}\end{array}$ & $\begin{array}{c}\boldsymbol{X}_{\mathbf{0}}, \\
\mathbf{O h m} / \\
\mathbf{k m}\end{array}$ & $\begin{array}{c}\boldsymbol{G}_{\mathbf{0}}, \\
\boldsymbol{\mu} \mathbf{S} / \mathbf{k m}\end{array}$ & $\begin{array}{c}\boldsymbol{B}_{\mathbf{0}}, \\
\boldsymbol{\mu} \mathbf{S} \mathbf{k m}\end{array}$ \\
\hline $\bar{P}$ & 16,996 & 146,057 & 13,039 & 2015,126 & 0,037 & 0,304 & 0,012 & 3,892 \\
\hline$\sigma(P)$ & 0,305 & 0,308 & 0,910 & 0,886 & 0,001 & 0,001 & 0,002 & 0,002 \\
\hline$\frac{\sigma(P)}{\bar{P}} \cdot 100, \%$ & 1,797 & 0,211 & 6,980 & 0,044 & 1,792 & 0,222 & 13,892 & 0,044 \\
\hline$\frac{\left(\bar{P}-P_{N}\right)}{P_{N}} \cdot 100, \%$ & 0,325 & 0,022 & 0,614 & $-0,056$ & 0,322 & 0,020 & 1,015 & $-0,055$ \\
\hline
\end{tabular}

Table 3. The results of estimation of line parameters within using the sum of modules of correlation coefficients as an objective function

\begin{tabular}{|c|c|c|c|c|c|c|c|c|}
\hline Parameter & $\begin{array}{c}\boldsymbol{R}, \\
\mathbf{O h m}\end{array}$ & $\begin{array}{c}\boldsymbol{X}, \\
\mathbf{O h m}\end{array}$ & $\begin{array}{c}\boldsymbol{G}, \\
\boldsymbol{\mu S}\end{array}$ & $\begin{array}{c}\boldsymbol{B}, \\
\boldsymbol{\mu S}\end{array}$ & $\begin{array}{c}\boldsymbol{R}_{\mathbf{0}}, \\
\mathbf{O h m} /\end{array}$ & $\begin{array}{c}\boldsymbol{X}_{\mathbf{0}}, \\
\mathbf{~ \mathbf { h m }} / \\
\mathbf{k m}\end{array}$ & $\begin{array}{c}\boldsymbol{G}_{\mathbf{0}}, \\
\boldsymbol{\mu S} / \mathbf{k m}\end{array}$ & $\begin{array}{c}\boldsymbol{B}_{\mathbf{0}}, \\
\boldsymbol{\mu S} / \mathbf{k m}\end{array}$ \\
\hline $\bar{P}$ & 16,979 & 146,023 & 12,787 & 2016,006 & 0,037 & 0,304 & 0,012 & 3,894 \\
\hline$\sigma(P)$ & 0,305 & 0,308 & 0,910 & 0,885 & 0,001 & 0,001 & 0,002 & 0,002 \\
\hline$\frac{\sigma(P)}{\bar{P}} \cdot 100, \%$ & 1,799 & 0,211 & 7,118 & 0,044 & 1,795 & 0,222 & 14,443 & 0,044 \\
\hline$\frac{\left(\bar{P}-P_{N}\right)}{P_{N}} \cdot 100, \%$ & 0,222 & $-0,001$ & $-1,331$ & $-0,012$ & 0,217 & $-0,002$ & $-2,834$ & $-0,012$ \\
\hline
\end{tabular}


As follows from the results, using as an objective function the sum of squares of deviations and capacitive resistances error conductivity above and below for the conductance of similar errors, obtained by using as a objective function of the correlation coefficients. It can be assumed that the higher error of active conductivity in the second case can be explained by the exclusion from the objective function of the correlation coefficient between $G_{0}$ and $U_{1}^{2}+U_{2}^{2}$.

Table 4 show a comparison of the optimal values of RCFS at a different objective functions.

Table 4. The results of calculation of the RCFS within different objective functions

\begin{tabular}{|c|c|c|c|}
\hline Parameter & Reference & $\begin{array}{c}\text { Objective function is the sum } \\
\text { of squares of deviations }\end{array}$ & $\begin{array}{c}\text { Objective function is the sum of the } \\
\text { modules of the correlation coefficients }\end{array}$ \\
\hline $\mathrm{K}(\mathrm{I} 1), \%$ & 100,080 & 100,080 & 100,080 \\
\hline $\mathrm{K}(\mathrm{I} 2) \%$ & 100,120 & 100,103 & 100,112 \\
\hline $\mathrm{K}(\mathrm{U} 1), \%$ & 99,900 & 99,900 & 99,900 \\
\hline $\mathrm{K}(\mathrm{U} 2), \%$ & 100,050 & 100,060 & 100,055 \\
\hline $\mathrm{K}(\mathrm{dI} 1){ }^{\circ}$ & 0,400 & 0,420 & 0,405 \\
\hline $\mathrm{K}(\mathrm{dI} 2){ }^{\circ}$ & 0,200 & 0,180 & 0,201 \\
\hline $\mathrm{K}(\mathrm{dU} 1){ }^{\circ}$ & 0,000 & 0,000 & 0,000 \\
\hline $\mathrm{K}(\mathrm{dU} 2){ }^{\circ}$ & $-0,200$ & $-0,195$ & $-0,200$ \\
\hline
\end{tabular}

It follows from the presented results that all 5 estimated RCFS coefficients are more accurate when using the sum of the modules of the correlation coefficients as the objective function.

\subsection{The results of estimation of the line parameters by real measurements}

Similar calculations were made using real data from PMUs installed at the ends of the $500 \mathrm{kV}$ line. The results of estimation of the line parameters within different objective functions are shown in Tables 5 and 6, the results of calculation of the RCFS coefficients are shown in Table 7.

Table 5. The results of estimation of the line parameters by real measurements within using the sum of standard deviations as an objective function

\begin{tabular}{|c|c|c|c|c|c|c|c|c|}
\hline Parameter & $\begin{array}{c}\boldsymbol{R}, \\
\mathbf{O h m}\end{array}$ & $\begin{array}{c}\boldsymbol{X}, \\
\mathbf{O h m}\end{array}$ & $\begin{array}{c}\boldsymbol{G}, \\
\boldsymbol{\mu S}\end{array}$ & $\begin{array}{c}\boldsymbol{B}, \\
\boldsymbol{\mu S}\end{array}$ & $\begin{array}{c}\mathbf{R} \mathbf{0}, \\
\mathbf{\mathbf { S m }} /\end{array}$ & $\begin{array}{c}\boldsymbol{X}_{\mathbf{0}}, \\
\mathbf{O h m} / \\
\mathbf{k m}\end{array}$ & $\begin{array}{c}\boldsymbol{G}_{\mathbf{0}}, \\
\boldsymbol{\mu} \mathbf{S} / \mathbf{k m}\end{array}$ & $\begin{array}{c}\boldsymbol{B}_{\mathbf{0}}, \\
\boldsymbol{\mu S} / \mathbf{k m}\end{array}$ \\
\hline $\bar{P}$ & 16,390 & 146,522 & $-5,188$ & 2010,501 & 0,036 & 0,305 & $-0,021$ & 3,883 \\
\hline$\sigma(P)$ & 0,096 & 0,100 & 0,622 & 1,048 & 0,000 & 0,000 & 0,001 & 0,002 \\
\hline$\frac{\sigma(P)}{\bar{P}} \cdot 100, \%$ & 0,586 & 0,069 & 11,982 & 0,052 & 0,584 & 0,073 & 5,562 & 0,051 \\
\hline$\frac{\left(\bar{P}-P_{N}\right)}{P_{N}} \cdot 100, \%$ & $-3,255$ & 0,340 & $-140,032$ & $-0,285$ & $-3,622$ & 0,360 & $-274,171$ & $-0,293$ \\
\hline
\end{tabular}

Table 6. The results of estimation of the line parameters by real measurements within using the sum of modules of correlation coefficients as an objective function

\begin{tabular}{|c|c|c|c|c|c|c|c|c|}
\hline Parameter & $\begin{array}{c}\boldsymbol{R}, \\
\mathbf{O h m}\end{array}$ & $\begin{array}{c}\boldsymbol{X}, \\
\mathbf{O h m}\end{array}$ & $\begin{array}{c}\boldsymbol{G}, \\
\boldsymbol{\mu S}\end{array}$ & $\begin{array}{c}\boldsymbol{B}, \\
\boldsymbol{\mu S}\end{array}$ & $\begin{array}{c}\boldsymbol{R}_{\mathbf{0}}, \\
\mathbf{O h m} / \\
\mathbf{k m}\end{array}$ & $\begin{array}{c}\boldsymbol{X}_{\mathbf{0}}, \\
\mathbf{O h m} / \\
\mathbf{k m}\end{array}$ & $\begin{array}{c}\boldsymbol{G}_{\mathbf{0}}, \\
\boldsymbol{\mu S} / \mathbf{k m}\end{array}$ & $\begin{array}{c}\boldsymbol{B}_{\mathbf{0}}, \\
\boldsymbol{\mu S} / \mathbf{k m}\end{array}$ \\
\hline $\bar{P}$ & 17,677 & 147,660 & 16,998 & 2019,507 & 0,039 & 0,308 & 0,019 & 3,900 \\
\hline$\sigma(P)$ & 0,202 & 0,101 & 0,633 & 0,727 & 0,000 & 0,000 & 0,001 & 0,001 \\
\hline$\frac{\sigma(P)}{\bar{P}} \cdot 100, \%$ & 1,143 & 0,068 & 3,722 & 0,036 & 1,139 & 0,072 & 6,241 & 0,035 \\
\hline$\frac{\left(\bar{P}-P_{N}\right)}{P_{N}} \cdot 100, \%$ & 4,345 & 1,120 & 31,165 & 0,161 & 4,568 & 1,179 & 56,973 & 0,129 \\
\hline
\end{tabular}


Table 7. The results of calculation of the RCFS by real measurements within different objective functions

\begin{tabular}{|c|c|c|}
\hline Parameter & $\begin{array}{c}\text { Objective function - } \\
\text { sum of squares } \\
\text { of deviations }\end{array}$ & $\begin{array}{c}\text { Objective function - the sum } \\
\text { of the modules of the } \\
\text { correlation coefficients }\end{array}$ \\
\hline $\mathrm{K}(\mathrm{I} 1), \%$ & 100.000 & 100.000 \\
\hline $\mathrm{K}(\mathrm{I} 2), \%$ & 100.256 & 100.188 \\
\hline $\mathrm{K}(\mathrm{U} 1), \%$ & 100.000 & 100.000 \\
\hline $\mathrm{K}(\mathrm{U} 2), \%$ & 99.600 & 100.006 \\
\hline $\mathrm{K}(\mathrm{dI} 1){ }^{\circ}$ & 0.999 & -0.050 \\
\hline $\mathrm{K}(\mathrm{dI} 2){ }^{\circ}$ & 0.375 & 0.000 \\
\hline $\mathrm{K}(\mathrm{dU} 1){ }^{\circ}$ & 0.000 & 0.000 \\
\hline $\mathrm{K}(\mathrm{dU} 2){ }^{\circ}$ & 0.113 & 0.165 \\
\hline
\end{tabular}

As can be seen from the presented results, the resistance estimation errors in the first case are less than in the second. However, the error in determining the active conductivity has an unacceptable value and contradicts the physical meaning of the problem being solved, since the active conductivity is negative. A more detailed analysis of the results showed that with negative and lower modulus of active conductivity, both resistance and capacitive conductivity have less than the nominal values. Accordingly, the standard deviations are also smaller, which corresponds to the objective function in this calculation. When the sum of the modules of the correlation coefficients is used as an objective function, this drawback is absent.

The specific active conductivity is positive and is $57 \%$ higher than the nominal value for good weather. This result correlates well with the increased air humidity during the study period along the overhead line. The deviation of $\mathrm{B}_{0}$ from the nominal value is only $0.13 \%$, compared with $-0.29 \%$ when using the sum of squares of deviations as an objective function. It should also be noted that the coefficients of variation $\mathrm{X}_{0}$ and $\mathrm{B}_{0}$ have smaller values: $0.72 \%$ and $0.035 \%$, respectively, versus $0.73 \%$ and $0.051 \%$.

The ability to correctly evaluate the active conductivity of the $500 \mathrm{kV}$ overhead line makes it possible to optimize its operation in order to reduce energy losses. So for the investigated overhead line, optimization calculations showed that due to the optimal regulation of reactive power flows and voltages, the losses of active energy could be reduced from 12.347 thousand $\mathrm{kWh}$ to 12.029 thousand $\mathrm{kWh}$, i.e. by $2.5 \%$ in just one observed hour due to an increase in reactive power consumption by an average of 29.5 Mvar at the receiving end of the line without changing the voltage at its supply end. The averaged parameters of the line power flow for the investigated hour before and after optimization are shown in Table 8.

Table 8. Comparison of the transmission line $500 \mathrm{kV}$ power flows before and after optimization

\begin{tabular}{|c|c|c|c|}
\hline Parameters & before optimization & after optimization & Parameter changing \\
\hline $\mathrm{U} 1, \mathrm{kV}$ & 507,380 & 507,380 & 0 \\
\hline $\mathrm{U} 2, \mathrm{kV}$ & 517,243 & 505.262 & 11.981 \\
\hline $\mathrm{P} 1,{ }^{\circ} \mathrm{MW}$ & 338,277 & 338,277 & 0 \\
\hline $\mathrm{Q} 1, \mathrm{Mvar}{ }^{\circ}$ & -300.779 & $-259,948$ & -40.831 \\
\hline $\mathrm{P} 2{ }^{\circ} \mathrm{MW}$ & 325,930 & 326.248 & -0.318 \\
\hline $\mathrm{Q} 2, \mathrm{Mvar}^{\circ}$ & 163,340 & 192.814 & -29.474 \\
\hline $\mathrm{dP}_{\text {total }}, \mathrm{MW}^{\circ}$, incl. & 12,347 & 12.029 & 0.318 \\
\hline $\mathrm{dP}_{\mathrm{R}}, \mathrm{MW}^{\circ}$ & 7.885 & 7.770 & 0.115 \\
\hline $\mathrm{dP}_{\mathrm{G}}, \mathrm{MW}^{\circ}$ & 4.462 & 4.259 & 0.203 \\
\hline
\end{tabular}


Optimization of the power flow was performed for every second of the investigated hour interval. The voltage and active power flux for the supply end of the line (node 1) were taken the same as in the initial power flow. The optimal value of the reactive power flux at the receiving end of the line was chosen so that in the $\pi$-equivalent circuit of the transmission line, the reactive power flux at the beginning of the branch was zero. It is not difficult to show that at a given voltage at the transmitting end of the line, this power flow corresponds to the minimum power loss in the longitudinal resistance of the equivalent circuit. As follows from the results presented in Table 8, the average reduction in power losses in the longitudinal resistance was $0.115 \mathrm{MW}$ or $1.5 \%$. By increasing the reactive power in the optimal power flow at the receiving end of the line by an average of 29.5 Mvar, the voltage at the receiving end of the line decreased to $502.3 \mathrm{kV}$. This led to a decrease in active power losses in the transverse conductivity of node 2 of the $\pi$-equivalent circuit of the overhead line by an average of $0.203 \mathrm{MW}$ or $4.5 \%$ of the active losses in conductivity in the initial power flow. When calculating the optimal overhead line power flow, the dependence of losses in conductivity was modelled by a fourth-order power function. As mentioned above, corona losses depend on the voltage. The exponent of dependence is in the range of 4-10. Calculations show that at powers of 10 , the reduction in transverse conduction losses is $0.473 \mathrm{MW}$.

\subsection{Recommendations to improve the accuracy of estimation of transmission line parameters with PMU}

To improve the accuracy of the simultaneous estimation of the parameters of overhead lines and RCFS, it is advisable in future studies to take into account the following points:

1. The values of the parameters $X_{0}, B_{0}$ weakly dependent on the power flow, air temperature and other weather phenomena. Therefore, for them it is proposed to use narrow intervals of the minimum $\left(X_{0 \min }, B_{0 \min }\right)$ and maximum $\left(X_{0 \max }, B_{0 \max }\right)$ values to be taken into account in the penalty function.

2. The active resistance of an overhead line is linearly dependent on temperature. Taking into account the local climate, it is possible to roughly estimate the possible limits of changes in the active resistance $R_{\min }, R_{\max }$ to be taken into account in the penalty function. As a rule, this interval is less than the value of $\pm 15 \%$ from the nominal value of $R$.

3. Corona losses cannot be less than a certain positive value and more than the maximum value with frost. After the minimum and maximum voltage of transmission line can be calculated are marginal values $G_{0}$, are taken into account by means of the free function (6) in the objective function.

\section{Conclusions}

An improved method for estimation of transmission lines parameters with PMU is proposed. Its advantages are shown in comparison with existing methods on the example of artificially modelled data and on the basis of measurements from existing PMUs.

For artificially modeled data, all 5 estimated KFCS coefficients turned out to be closer to the reference values when using the sum of the moduli of the correlation coefficients as an objective function. Accordingly, 3 out of 4 estimated specific parameters of transmission lines turned out to be closer to the standards.

The results according to PMU data for real studies of $500 \mathrm{kV}$ transmission lines with a length of $504.643 \mathrm{~km}$ showed that when using the sum of the modules of the correlation coefficients as a function of the sum function, the deviation of $B_{0}$ from the nominal value is 
only $0.13 \%$, compared with $0.29 \%$ when used in use as a function of the sum of squared deviations. The coefficients of variation $X_{0}$ and $B_{0}$ have smaller values: $0.72 \%$ and $0.035 \%$, respectively, versus $0.73 \%$ and $0.051 \%$. In addition, when the sum of squares of deviations is used as an objective function, the estimated value of active conductivity has a negative sign, which contradicts the physical meaning of the problem being solved.

Optimization calculations showed that due to the optimal regulation of the flow power and voltages based on the estimated parameters of the transmission line, losses in it in one hour could be reduced by $2.5 \%$ from 12.35 thousand $\mathrm{kWh}$ to 12.03 thousand $\mathrm{kWh}$ while maintaining the initial voltage at the transmitting end of the line. In the presence of technical capabilities to reduce the voltage and at the transmitting end of the line, the relative decrease in the total losses of the transmission line can reach several tens of percent during inclement weather and increased corona losses.

Recommendations are given for improving the accuracy of identification of the parameters of transmission lines, which consist in taking into account the two-sided restrictions on the estimated parameters in the objective function in the form of penalties.

This work was supported by JSC «STC FGC UES» in the framework of R\&D on the topic «Software and hardware complex of the automated system for optimizing the mode of MES South for voltage and reactive power».

\section{References}

1. T. Joseph, C. E. Ugalde-Loo, J. Liang and P. F. Coventry, Asset Management Strategies for Power Electronic Converters in Transmission Networks: Application to Hvdc and FACTS Devices, in IEEE Access, 6, 21084-21102 (2018). doi: 10.1109/ACCESS.2018.2826360.

2. Knopf, F. Modeling, Analysis and Optimization of Process and Energy Systems. (2011). doi:10.1002/9781118121160.

3. H. Goklani, G. Gajjar and S. A. Soman, Instrument Transformer Calibration and Robust Estimation of Transmission Line Parameters Using PMU Measurements, in IEEE Transactions on Power Systems, 36, 3, 1761-1770, (2021). doi: 10.1109/TPWRS.2020.3036605.

4. Y.G. Kononov, O. Rybasova, and K. Sidirov, Identification of overhead line parameters from PMU data with compensation of systematic measurement errors, in Proc. Int. Conf. Ind. Eng., Appl. Manuf., (2018)

5. M. M. Adibi and R. J. Kafka, Minimization of uncertainties in analog measurements for use in state estimation, in IEEE Transactions on Power Systems, 5, 3, 902-910, (1990). doi: 10.1109/59.65920.

6. K. Dasgupta and S. Soman, Line parameter estimation using phasor measurements by the total least squares approach, in Proc. Power Energy Soc. Gen. Meet., IEEE, Jul. 2013, pp. 1-5. (2013)

7. C. Mishra, V. A. Centeno, and A. Pal, Kalman-filter based recursive regression for three-phase line parameter estimation using synchrophasor measurements, in Proc. Power Energy Soc. Gen. Meet., IEEE, Jul. 2015, 1-5. (2015)

8. M. Asprou and E. Kyriakides, Estimation of transmission line parameters using PMU measurements, in Proc. Power Energy Soc. Gen. Meet., IEEE, Jul. 2015, 1-5. (2015)

9. H. Goklani, G. Gajjar, and S. A. Soman, Quantification of minimum unbalance required for accurate estimation of sequence parameters of transmission line using PMU data, in Proc. IEEE Power Energy Soc. Gen. Meet., Aug. 2019, 1-5. (2019) 
10. Z.Wu, L. Zora, and A. Phadke, Simultaneous transmission line parameter and PMU measurement calibration, in Proc. IEEE Power Energy Soc. Gen. Meet., Jul. 2015. 1-5. (2015)

11. K.V. Khandeparkar, S.A. Soman, and G. Gajjar, Detection and correction of systematic errors in instrument transformers along with line parameter estimation using PMU data, IEEE Trans. Power Syst., 32. 4, 3089-3098. (2017)

12. Yu.G. Kononov, O.S. Rybasova, and S.S. Kostyukova, Determination Eqiuvalent circuit parameters overhead line $750 \mathrm{kV}$ according to vector registers, Digests 6th Int. Scientific and Technical Conf., (2015)

13. Deborah Ritzmann; Paul S. Wright; William Holderbaum; Ben Potter, A Method for Accurate Transmission Line Impedance Parameter Estimation, IEEE Transactions on Instrumentation and Measurement, 65, 10, 2204 - 2213 (2016)

14. Deborah Ritzmann; Johan Rens; Paul S. Wright; William Holderbaum; Ben Potter, $A$ Novel Approach to Noninvasive Measurement of Overhead Line Impedance Parameters, IEEE Transactions on Instrumentation and Measurement, 66, 6, 1155 - 1163 (2017)

15. Swaroop Gajare, Ashok Kumar Pradhan, Vladimir Terzija, A Method for Accurate Parameter Estimation of Series Compensated Transmission Lines Using Synchronized Data, IEEE Transactions On Power Systems, 32, 6, 4843 - 4850 (2017)

16. Hemantkumar Goklani, Gopal Gajjar, S. A. Soman, Instrument Transformer Calibration and Robust Estimation of Transmission Line Parameters Using PMU Measurements, IEEE Transactions On Power Systems, 36, 3, 1761 - 1770 (2021)

17. I.E. Ivanov, Bulletin of Ivanovsk state energy university, 1, 30-42 (2019)

18. P.I.Bartolomey, S.A. Eroshenko, E.M. Lebedev, A.A. Suvorov, New information technologies for state estimation of power systems with facts. Proc. 3rd IEEE PES Innovative Smart Grid Technologies Europe (ISGT Europe). Berlin, Germany, Oct. 14-17, (2012)

19. Diachenko A., Hussein A., Kononov Y., Sidorov K., Investigation of online corona losses as a function of operating voltage based on PMU data, 16th international conference on electrical machines, drives and power systems, Elma (2019)

20. A. Balametov, E. Halilov and T. Isayeva, Extra high voltage transmission line operation simulation using the actual corona-loss characteristics, Turkish Journal of Electrical Engineering \& Computer Sciences, 479-488, (2018)

21. A.D. Diachenko, Yu.G. Kononov, O. S. Rybasova, A.V. Petrov, Online Corona Losses Investigation During Overcast In Real 500 KV TLS Based On PMU Data, 2019 2nd International Youth Scientific and Technical Conference on Relay Protection and Automation (RPA) (2019)

22. V. A. Venikov, Electrical systems. Volume 2. Electrical networks, Moscow, (1971) 